\title{
Selecting Informative Actions Improves Cooperative Multiagent Learning
}

\author{
Liviu Panait \\ George Mason University \\ 4400 University Drive MSN 4A5 \\ Fairfax, VA 22030, USA \\ Ipanait@cs.gmu.edu
}

\author{
Sean Luke \\ George Mason University \\ 4400 University Drive MSN 4A5 \\ Fairfax, VA 22030, USA \\ sean@cs.gmu.edu
}

\begin{abstract}
In concurrent cooperative multiagent learning, each agent simultaneously learns to improve the overall performance of the team, with no direct control over the actions chosen by its teammates. An agent's action selection directly influences the rewards received by all the agents, resulting in a co-adaptation among the concurrent learning processes. Co-adaptation can drive the team towards suboptimal solutions because agents tend to select those actions that are rewarded better, without any consideration for how such actions may affect the search of their teammates. We argue that to counter this tendency, agents should also prefer actions that inform their teammates about the structure of the joint search space in order to help them choose from among various action options. We analyze this approach in a cooperative coevolutionary framework, and we propose a new algorithm, iCCEA, that highlights the advantages of selecting informative actions. We show that iCCEA generally outperforms other cooperative coevolution algorithms on our test problems.
\end{abstract}

\section{Categories and Subject Descriptors}

\section{I.2.11 [Distributed Artificial Intelligence]: Multiagent Systems}

\section{General Terms}

Experimentation

\section{Keywords}

Multiagent Learning, Cooperation, Coordination, Cooperative Coevolution

\section{INTRODUCTION}

Multiagent learning is challenging because the problem dynamics are often complex and fraught with local optima. Of particular interest to us is cooperative multiagent learning, where multiple agents learn to work together as a team to accomplish common goals [19]. More specifically, we are interested in concurrent learning, where each agent performs its own learning and has little or no

Permission to make digital or hard copies of all or part of this work for personal or classroom use is granted without fee provided that copies are not made or distributed for profit or commercial advantage and that copies bear this notice and the full citation on the first page. To copy otherwise, to republish, to post on servers or to redistribute to lists, requires prior specific permission and/or a fee.

AAMAS'06 May 8-12 2006, Hakodate, Hokkaido, Japan.

Copyright 2006 ACM 1-59593-303-4/06/0005 ...\$5.00. control over the other agents' selection of actions.

Multiagent learning is problematic for existing machine learning techniques because the concurrent learning processes are not independent. Consider an agent that observes an environment containing other agents and that tries to improve its performance. This leads to a modification in its behavior, which is then sensed by the other agents, who then change their behaviors in order to improve their performance as well. This "moves the goalpost" on the original agent: its newly-learned behavior may no longer be appropriate. Thus as the agents co-adapt to one another, the environment is essentially changing beneath the agents' feet. Moreover, the agent itself contributes directly to how the landscape changes. Learning in the face of this dynamic is not easy: such co-adaptation can result in cyclical or chaotic adaptive behavior, and may gravitate towards suboptimal solutions.

Most cooperative multiagent learning algorithms assume the agents are rational: each agent searches for actions that fare well when used in combination with the actions currently favored by its teammates. This "best-response" approach usually results in the learners converging to Nash equilibria. Such "rational" convergence to equilibria may well be movement away from globally team-optimal solutions $[16,26]$. To counter this, we argue that agents must also explore actions that inform their teammates about the structure of the space of rewards for joint actions. For example, if an agent identifies an action that helps other agents rank their available actions better, the agent should explore that action to help guide the teammates' learning processes.

Though we believe it to be general, we will demonstrate the application of this approach to a particular multiagent learning method of interest to us, namely cooperative coevolution $[12,25]$. Coevolution is the use of evolutionary computation learning techniques in a multiagent setting. Ordinarily, evolutionary computation employs only a single learner to discover a global solution to an optimization problem: the learner first creates an initial pool of randomly-generated candidate solutions (a "population" of "individuals"), then assesses their quality ("fitness") independently of one another, then forms a new population of individuals through iteratively selecting, copying, and modifying ("breeding") individuals from the previous population with an emphasis on the fitter members of that previous population. The new population replaces the old one, and this cycle of fitness assessment, breeding, and population replacement continues until a sufficiently fit individual is discovered or until resources have expired. Each iteration of this cycle is known as a "generation".

Cooperative coevolutionary algorithms (CCEAs), in the form we will discuss here, use not one but multiple populations, each involved in its own separate learning cycle of fitness assessment, population formation, and population replacement. However, indi- 
viduals in a given population are no longer assessed independently, but rather in the context of individuals chosen from the other populations. Each population represents a sub-component of a full solution to the problem, and as part of its fitness assessment, an individual in a given population may be evaluated only by combining it with one individual from each of the other populations to form a complete solution. It is in this fashion that coevolution involves multiple learners (each of the populations' evolutionary search procedures) whose learning trajectories are intertwined (via joint fitness evaluation), and so coevolution must deal with the same coadaptation challenges as other multiagent learning methods.

A natural approach to applying CCEAs to cooperative multiagent learning is to assign one population to each of the learning agents in the team. Each individual in the population represents a potential behavior for the agent, and so from now on, for consistency, we will refer to actions rather than individuals. An action may be as simple as a single action in trivial environments, or as complex as policies involving internal states and memory for realworld problems. Thus each population represents a finite sample from an infinite space of possible actions. As the team reward permits only the evaluation of joint actions, an action in one agent's population may be evaluated when combined with actions from the other agents' current populations. Multiple such combinations are generally used.

Section 2 highlights related cooperative multiagent learning algorithms. Following, Section 3 introduces a novel learning algorithm where agents pay special attention to informative actions. We compare it against other cooperative multiagent learning techniques in Section 4. The paper concludes with a brief discussion of our findings, accompanied by directions for future work.

\section{RELATED WORK}

Two learning algorithms that are guaranteed to find the globally optimal joint action in a stateless environment are proposed in [4]. Both algorithms have two phases: agents first explore the entire space of joint actions (either deterministically or randomly); this is followed by a greedy selection of only the action (one per agent) that returned the highest reward. Both algorithms find the global optimum in polynomial time in the number of actions for each agent. However, scaling these algorithms to environments with states or with possibly infinite numbers of actions per agent may be problematic.

Instead of choosing actions deterministically or randomly, Claus and Boutilier [8] argue that agents should be more optimistic about their teammates: an agent should not prefer actions that do well in the context of the actions currently preferred by its teammates, but rather the agent should prefer actions that do well in the context of better actions that its teammates might learn. The application of this heuristic assumption results in additional multiagent reinforcement learning algorithms for stateless environments, such as the ones in $[15,14]$. Research on scaling these algorithms from simple coordination games to more complex domains is reported in $[3,11$, $18,27]$.

The cooperative coevolution literature has followed a similar path. The properties of cooperative coevolutionary algorithms are analyzed in $[6,7,29]$; results of such experiments indicate that assessing the fitness of an action based on the maximum of multiple joint rewards works better than if it were based on the minimum or on the average. Recent work has analyzed the conditions under which coevolutionary systems gravitate towards suboptimal solutions [28], has provided a visual illustration of the basins of attraction for simple multiagent domains [23], and has proposed a biased CCEA that is more likely to find the global optimum [21].
Bucci and Pollack [5] apply recent advances from competitive coevolution research to improve CCEAs, resulting in the PCCEA algorithm. The authors argue that the aggregation of multiple joint rewards to compute the fitness of an action may result in loss of useful information. Instead, pCCEA uses all joint rewards to compute the set of actions in each population that are Pareto non-dominated: given two actions $a_{1}$ and $a_{2}$ for one agent, $a_{1}$ dominates $a_{2}$ if and only if (1) for any action $b$ for the other agent, $a_{1}$ receives higher or equal reward when joined with $b$ than $a_{2}$ does, and (2) there exists an action $c$ for the other agent such that $a_{1}$ receives a strictly higher reward when joined with $c$ than $a_{2}$ does. This set of nondominated actions, termed an archive, is automatically copied to the next generation to help evaluate the new population of actions. After the evaluation is completed for that generation, a new archive is computed for each population. Our experiments in Section 4 indicate that pCCEA's archive tends to converge to the Pareto frontier, which unfortunately may be infinite in even simple cooperative multiagent domains.

\section{THE iCCEA ALGORITHM}

When multiple agents learn concurrently, each of them is afforded only a partial glimpse at the overall search space. Specifically, each agent may weight its actions using only a projection of the entire space, a projection that is influenced by the actions currently chosen by the other agents according to their own learning processes. Differences among such projections are illustrated in [20]: projections at early stages of learning may provide more information about the search space, because the agents' actions are more randomly distributed and so sample the joint space better. As the agents start to converge, the projections may become skewed, sometimes losing all information about the globally optimal solutions. As each agent's choice of actions influences the projections searched by the other agents, we may view each of the multiagent learning algorithms in Section 2 as recipes for agents to influence each other's learning processes.

We argue that the team of learning agents may benefit if each agent is concerned about the projection of the search space that it provides to its teammates via the actions it explores. In other words, agents should not necessarily explore only their most promising actions, but also those actions that provide the other agents with accurate projections of the joint search space. We propose a coevolutionary algorithm, iCCEA ("i" standard for "informative"), to illustrate the advantages of such an approach.

For simplicity, we present the pseudocode for the algorithm using only two agents (and hence two populations), although it may be extended to arbitrary numbers of agents. Given an agent, the other agent is referred to as its teammate. We will assume that all populations have equal size PopSize, though the algorithm can easily be extended to allow for different sizes. We define $\operatorname{Reward}_{p}(i, a)$ as the reward received when an agent (whose actions are represented in population $p$ ) selects action $i$ and its teammate selects action $a$.

iCCEA follows the standard architecture of a generational cooperative coevolutionary algorithm [25]. As is the case for other CCEAs, iCCEA assumes that an agent can perceive the actions chosen by its teammates. In iCCEA, as in PCCEA, each agent maintains a population of actions, a subset of which is defined as an archive responsible for ensuring that some actions exist primarily to keep the teammate's projection well informed. iCCEA agents learn concurrently, meaning, the populations advance through their generation cycles together, rather than one population advancing, then the other. 
The evaluation process tests actions by combining them with actions from the teammate's archive, plus possibly some additional actions in the teammate's population. In addition to computing an action's fitness, the evaluation process stores the joint reward of any pair of actions that are evaluated together; this information is later used to update the archives of each agent.

Each generation, every action in a population is first evaluated by testing it in combination with every action from the teammate's archive. As the very first generation's populations have not built an archive yet, their entire population is used as an archive (an expensive process but one which ensures a thorough exploration of the joint space and good bootstrapping for the archive in future generations). If the maximum size of the archives is less than MaxEvals, the actions are also tested with enough randomly-chosen actions from the teammate's population (in the archive or not) to provide at least MaxEvals tests per action. MaxEvals establishes a threshold for the number of evaluations desired per each action: if the archive is too small, additional actions are sampled at random from the other population to provide additional assessments.

The fitness of action $i$ is then set to maximum of $F_{i}^{j}$ over all actions $j$ in the teammate's population $\left(F_{i}^{j}\right.$ represents the fitness of action $i$ when partnered with action $j$ from the other population, and it equals $-\infty$ if $i$ was not tested in combination with $j$ ). If the archive size is 1 , this reduces to a common evaluation approach for CCEAs [25, 29]: the evaluation is equivalent to using the best action (from the previous generation) plus some actions chosen at random from the other population. The pseudocode for the evaluation process is:

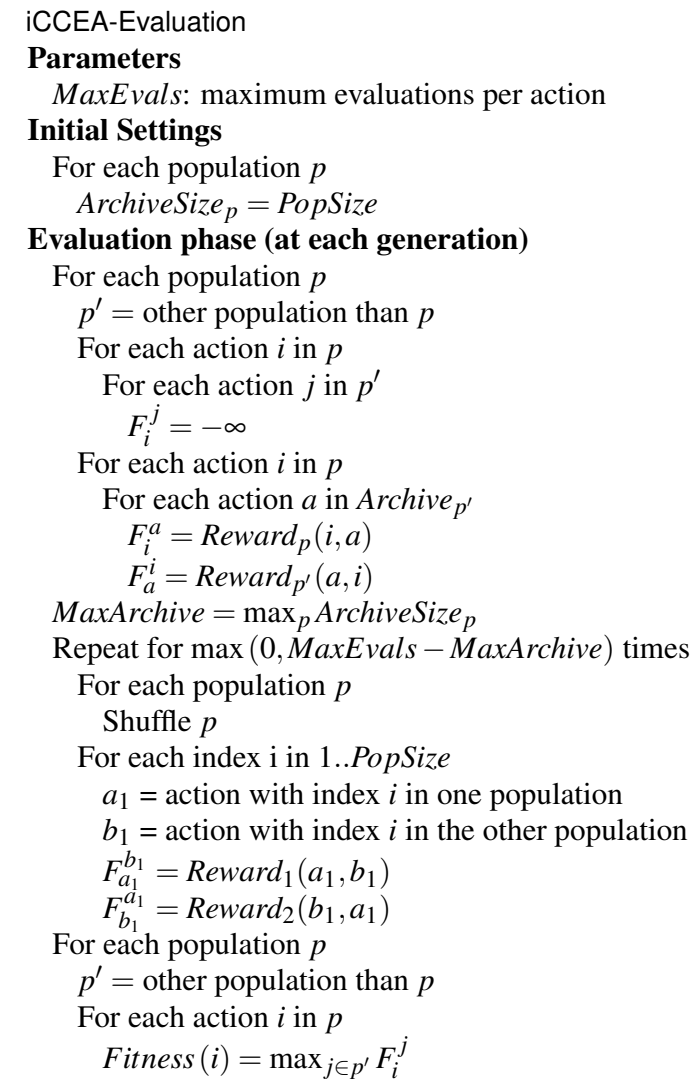

We next assemble the archive through a process called archive selection, which is intended to select those actions which revealed features of the projected joint space useful to the other teammate. Specifically, we would like to select as an archive a minimal set of actions from each agent's population such that when assessing the fitness of actions in a given population, testing them against the full teammate's population would not change the rank ordering of their fitnesses beyond just testing against the teammate's archive. The hope is that this archive would provide an accurate evaluation and ranking of the teammate's actions in the next generation as well. Rank order is all that matters because the evolutionary selection procedure we use later compares individuals not on raw fitness, but solely on their relative rank order in the population.

We'd like the archive set to be as small as possible, because as each action in an agent's population is tested in combination with every action from the other agent's archive, large archives imply a prohibitive $O\left(n^{2}\right)$ evaluation cost. Therefore we add actions to the archive only if they cause actions in the other population to improve significantly enough so as to effect the ranking — causing actions to worsen is not considered helpful information. Of the various actions which change this ranking, we will select the ones which do so by raising fitnesses to the highest levels.

The archive selection process starts from the empty set and proceeds iteratively. For each action $i$ not in an agent's archive, and for each action $x$ in its teammate's population, we compute the fitness of $x$ if evaluated in combination with all actions in the current archive, that is, $M 1 F i t_{x}=\max _{j \in \text { Archive }_{p}} F_{x}^{j}$. We also compute the fitness of $x$ if $i$ were added to the current archive, $M 2 F i t_{x}^{i}=\max _{j \in \text { Archive }_{p} \cup\{i\}} F_{x}^{j}$. Note that M2Fit $i_{x}^{i} \geq M 1 F i t_{x}$. Our first criterion for adding $i$ to the archive (it improves upon the current ordering of the teammate's population) translates into finding two actions $x$ and $y$ such that adding $i$ to the archive would change their ranking relative to one another $\left(M 1 F i t_{x} \leq M 1 F i t_{y}\right.$ and $M 2 F i t_{x}^{i}>M 2 F i t_{y}^{i}$, or equivalently $M 3 F i t_{x, y}^{i} \neq-\infty$ for a simpler shorter notation ). Of all actions $i$ that meet this first criterion, we greedily prefer the one that changed the ranking by raising the fitness of a teammate's action to the highest level. Note that the first action to be selected for the archive is always the action with the highest fitness. The pseudocode is:
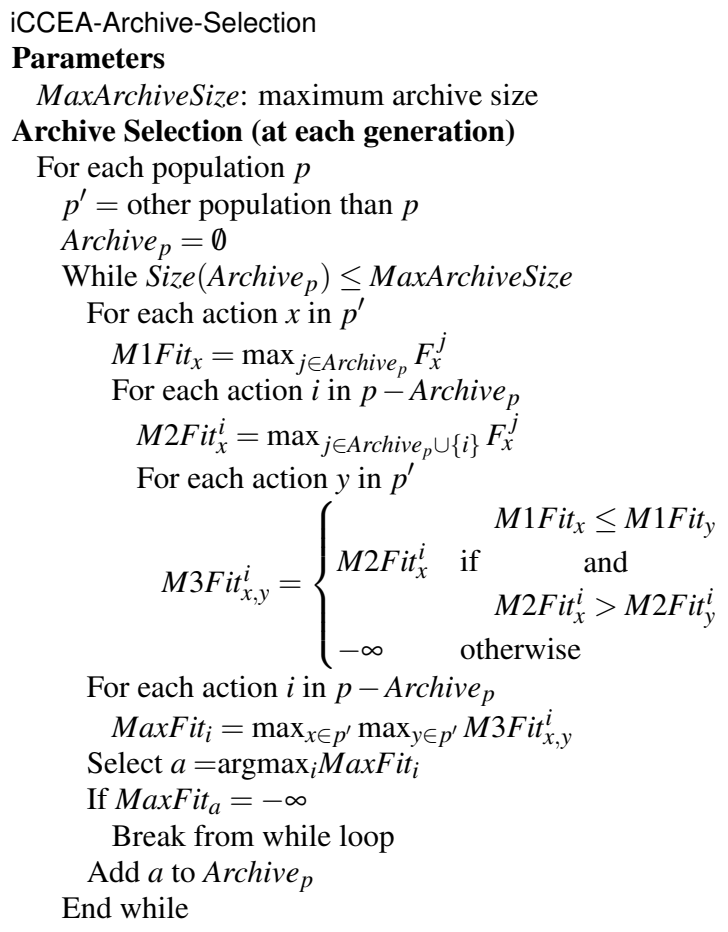
The breeding and population reassembly phase of iCCEA proceeds similarly to the one in PCCEA: the archive members are selected from the old population and are copied directly into the new population, and remainder of the new population is filled with children bred using standard evolutionary computation methods ${ }^{1}$ applied to the old population (including the old archive). The whole previous population (including the archive) competes for breeding. The pseudocode is straightforward:

\section{iCCEA-Breeding}

\section{Breeding phase (at each generation)}

For each population

Select its new archive with iCCEA-Archive-Selection

Copy the archive into the new population

Fill the remainder of the new population using standard EC breeding with a rank-based selection procedure (such as tournament selection)

\section{EXPERIMENTS}

In this section, we investigate the behavior of four different coevolutionary algorithms, and we compare them in terms of performance and in terms of the number of evaluations they require to achieve that performance. The first algorithm is the pCCEA algorithm introduced in [5]. Second is the cCCEA algorithm, which is a traditional CCEA algorithm that evaluates the fitness of an action as the maximum reward it receives when in combination with any of the actions in the teammate's population. cCCEA is guaranteed to converge to the global optimum if the population size is sufficiently large [21]. Third is the iCCEA algorithm proposed in Section 3. Fourth, rCCEA evaluates the fitness of an action as the maximum when partnered with six actions from the teammate's population: five chosen at random, plus the fittest action from the teammate's previous-generation population. We included this algorithm in the comparison because it uses a small fixed number of evaluations in each generation, such as is preferable for applications of multiagent learning to real problems.

We will test these algorithms using a class of problem domains called the maximum of two quadratics (or MTQ). These problems include a global optimum and a local suboptimum, where the suboptimum covers a much wider range of the search space and is thus difficult to escape. The problems have been used before by [5, 22].

We will assume that each action is a real-valued number from 0 to 1 inclusive. This defines a metric space for actions: in some sense action 0.5 is more similar to action 0.6 than action 0.9 is. While other techniques search for optima among sets of actions that have no "distance" relation among them, EC methods assume a distance relation: when breeding an action to form a new one, they will generally make more small (distance) changes than large changes.

The joint reward function for the MTQ class is defined as:

$$
\operatorname{MTQ}(x, y) \leftarrow \max \left\{\begin{array}{l}
H_{1} *\left(1-\frac{16 *\left(x-X_{1}\right)^{2}}{S_{1}}-\frac{16 *\left(y-Y_{1}\right)^{2}}{S_{1}}\right) \\
H_{2} *\left(1-\frac{16 *\left(x-X_{2}\right)^{2}}{S_{2}}-\frac{16 *\left(y-Y_{2}\right)^{2}}{S_{2}}\right)
\end{array}\right.
$$

where $x$ and $y$ may take values (actions) ranging between 0 and 1 . Different settings for $H_{1}, H_{2}, X_{1}, Y_{1}, X_{2}, Y_{2}, S_{1}$, and $S_{2}$ affect the difficulty of the problem domain in one of the following aspects. $H_{1}$ and $H_{2}$ affect the heights of the two peaks: higher peaks may increase the chance that the algorithm converges there. $S_{1}$ and $S_{2}$ affect the area that the two peaks cover: a higher value for one of

\footnotetext{
${ }^{1}[2,9]$ are good references for standard evolutionary algorithms.
}

them results in a wider coverage of the specific peak. This makes it more probable that the coevolutionary search algorithm will converge to this peak, even though it may be suboptimal. Different values for $X_{1}, Y_{1}, X_{2}$, and $Y_{2}$ result in changes in the locations of the centers of the two quadratics, which also affect the relatedness of the two peaks: similar values of the $x$ or $y$ coordinates for the two centers imply higher overlaps of the projections along one or both axes (the projections of the joint action space for one or both agents may retain more information about the globally optimal solution even if the other agent's population starts to converge to the suboptimal solution). In these experiments, we set $S_{1}=\frac{16}{10}, X_{1}=\frac{3}{4}$, $Y_{1}=\frac{3}{4}, H_{2}=150, S_{2}=\frac{1}{32}, X_{2}=\frac{1}{4}, Y_{2}=\frac{1}{4} ; H_{1}$ was varied across experiments, but it was always less than 125 .

MTQ using such settings is fairly difficult to optimize: the probability that a random sample has a function value within 0.01 of the optimal solution (which has a value of 150) can be computed as $\pi *\left(1-\frac{149.99}{150}\right) * \frac{S_{2}}{16}=0.0000004090615$. Given 51200 random samples (approximately the number of action evaluations performed during a typical evolutionary run), the probability that one of them exceeds a function value of 149.99 is $1-(1-0.0000004090615)^{51200}=0.02072615$.

As we will see in the next sections, the median of the results for the proposed iCCEA method is significantly higher than 149.99, which implies that iCCEA finds better approximations of the global optimum in more than $50 \%$ of the runs. This shows that iCCEA significantly outperforms random search in this domain, and implicitly the algorithms proposed in [4].

To further increase the difficulty of the problem domains with respect to the algorithms we analyze, we created a second class of problem domains, SMTQ, which is defined as:

$$
\operatorname{SMTQ}(x, y) \leftarrow \max \left\{\begin{array}{l}
H_{1} *\left(1-\frac{16 *\left(x_{1}^{r}-X_{1}\right)^{2}}{S_{1}}-\frac{12 *\left(y_{1}^{r}-Y_{1}\right)^{2}}{S_{1}}\right) \\
H_{2} *\left(1-\frac{16 *\left(x_{2}^{r}-X_{2}\right)^{2}}{S_{2}}-\frac{12 *\left(y_{2}^{r}-Y_{2}\right)^{2}}{S_{2}}\right)
\end{array}\right.
$$

where $x_{1}^{r}, y_{1}^{r}, x_{2}^{r}$, and $y_{2}^{r}$ are the original $x$ and $y$ values (which ranged between 0 and 1 ) rotated around the centers of the two peaks by $\frac{\pi}{4}$ :

$$
\begin{aligned}
& x_{1}^{r}=\left(x-X_{1}\right) * \cos \frac{\pi}{4}+\left(y-Y_{1}\right) * \sin \frac{\pi}{4}+X_{1} \\
& y_{1}^{r}=\left(x-X_{1}\right) * \cos \frac{\pi}{4}-\left(y-Y_{1}\right) * \sin \frac{\pi}{4}+Y_{1} \\
& x_{2}^{r}=\left(x-X_{2}\right) * \cos \frac{\pi}{4}+\left(y-Y_{2}\right) * \sin \frac{\pi}{4}+X_{2} \\
& y_{2}^{r}=\left(x-X_{2}\right) * \cos \frac{\pi}{4}-\left(y-Y_{2}\right) * \sin \frac{\pi}{4}+Y_{2}
\end{aligned}
$$

Observe that the two peaks have ellipsoid shapes aligned diagonally with the axes, as opposed to circular shapes in the MTQ problem domains. The two Nash equilibria from the MTQ class have now become an infinity of Nash equilibria in the SMTQ class. This creates an additional difficulty for the coevolutionary search. We used the same values for $H_{2}, X_{1}, Y_{1}, X_{2}, Y_{2}, S_{1}$, and $S_{2}$ as for the MTQ class.

The experiments used the ECJ package [17]. Each population contained 32 actions. cCCEA and rCCEA used elitism of size 1, meaning that the fittest individual in each population in the previous generation is automatically copied into the next generation's population. The entire archive survived automatically from one generation to the next for iCCEA and pCCEA. Unless stated otherwise, iCCEA used MaxEvals $=5$ and MaxArchiveSize $=\infty$. The 


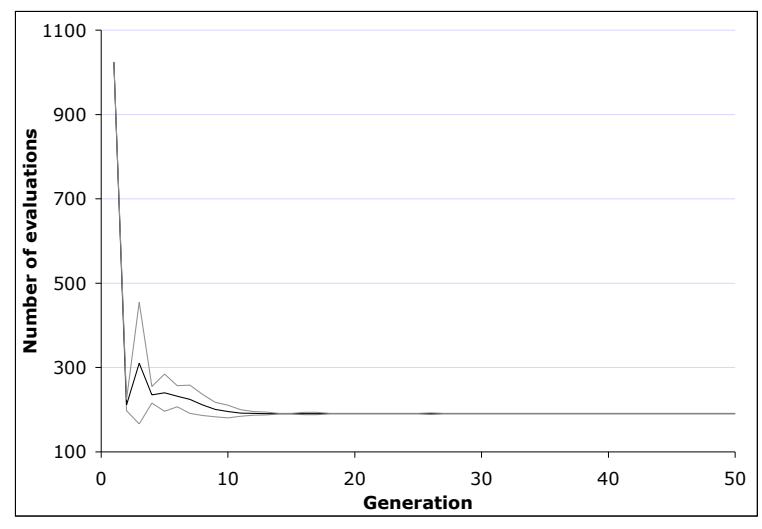

Figure 1: Average number of evaluations for the iCCEA algorithm in the MTQ domain instance with $\mathrm{H}_{1}=\mathbf{5 0}$

Table 1: Results of the four methods in the MTQ domain instance with $\mathrm{H}_{1}=\mathbf{5 0}$

\begin{tabular}{ccccc} 
Method & $\begin{array}{c}\text { First } \\
\text { Quartile }\end{array}$ & Median & $\begin{array}{c}\text { Third } \\
\text { Quartile }\end{array}$ & $\begin{array}{c}\text { Average } \\
\text { \# Evals }\end{array}$ \\
\hline pCCEA & 148.62776 & 149.74876 & 149.94931 & 51200 \\
cCCEA & 149.99971 & 149.99995 & 149.99998 & 51200 \\
iCCEA & 149.99990 & 149.99997 & 149.99998 & 10676.5 \\
rCCEA & 50 & 50 & 149.99998 & 19200
\end{tabular}

EC breeding method created children by selecting a parent, copying it, then "mutating" the copy by adding a gaussian random variable from a distribution with mean 0 and standard deviation 0.01 , bounding the value to between 0 and 1 . Parents were selected using "tournament selection", whereby two random parents are picked with replacement from the population, and the fitter of the two is selected. Runs lasted 50 generations.

The quality of a technique was defined as the average, over 250 independent runs, of the fitness of the best action (one per population) in the last generation of that run. The results usually have a peculiar bimodal distribution, with many values close to one of the two peaks. For this reason, we report information on the quartiles, as opposed to mean and standard deviation. For the same reason, we verify statistical significance via non-parametric $\mathrm{t}$-tests combined using the Bonferroni correction. Sometimes these non-parametric tests will return the opposite result than a regular parametric test would, and although some conclusions are different from the ones reported in [5], we believe our comparison methodology is well-founded.

\subsection{Experiment 1: MTQ and SMTQ, $\mathrm{H}_{1}=\mathbf{5 0}$}

In the first experiment, we set $H_{1}$ to 50 to have a wide difference between the height of the two peaks. In this case, coevolution may have difficulties finding the global optimum primarily because its coverage is significantly smaller than that of the suboptimal peak.

Table 1 presents the performance of the four methods in the MTQ instance $\left(H_{1}=50\right)$, as well as the number of evaluations required to achieve that performance. Although the differences appear small, the large number of observations (250) leads to statistically significant differences among the methods with confidence 95\%. The results indicate that rCCEA performs worst, followed by pCCEA, cCCEA and iCCEA. There are statistically significant dif-

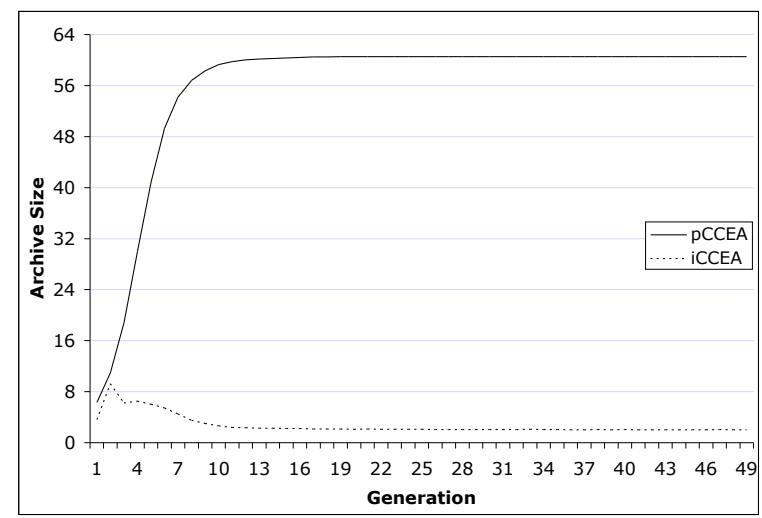

Figure 2: Average archive size for the iCCEA and the pCCEA algorithms in the MTQ domain instance with $\mathrm{H}_{1}=\mathbf{5 0}$

Table 2: Results of the four methods in the SMTQ domain instance with $\mathrm{H}_{1}=\mathbf{5 0}$

\begin{tabular}{ccccc} 
Method & $\begin{array}{c}\text { First } \\
\text { Quartile }\end{array}$ & Median & $\begin{array}{c}\text { Third } \\
\text { Quartile }\end{array}$ & $\begin{array}{c}\text { Average } \\
\text { \# Evals }\end{array}$ \\
\hline pCCEA & 133.21958 & 146.85126 & 149.49486 & 51200 \\
cCCEA & 149.99975 & 149.99995 & 149.99998 & 51200 \\
iCCEA & 149.99991 & 149.99997 & 149.99998 & 10982.34 \\
rCCEA & 50 & 149.99986 & 149.99998 & 19200
\end{tabular}

ferences between all pairs of methods. And importantly, iCCEA achieves a significant reduction in the number of evaluations as compared to the other methods.

Figure 1 plots the number of evaluations required by the iCCEA algorithm at each generation. The algorithm starts with a complete round-robin evaluation (requiring $32 \times 32$ evaluations), followed by a drastic decrease in the number of evaluations. This is due to the fact that the number of actions needed to accurately rank the other population (hence the archive size) decreases significantly. In contrast, in the pCCEA algorithm the Pareto front in the MTQ domain is not discrete, and thus the archive grows rapidly in size until it occupies the whole population (Figure 2). At this point, learning stagnates.

Table 2 presents the results of the four methods in the SMTQ domain, as well as the number of evaluations required to achieve that performance. The rCCEA and pCCEA methods perform worst (with no statistically significant difference among them). cCCEA is better than both rCCEA and pCCEA. iCCEA significantly outperforms all other methods. The dynamics of the archive size for PCCEA and iCCEA are very similar to those in the MTQ domain: pCCEA's archive again rises to consume most of the population, while iCCEA's archive size rises to 8 early but converges to approximately 5. This is summed over both populations. The average archive size for each population is half the value: an average size of 4 early on, and 2.5 later.

\subsection{Experiment 2: MTQ and SMTQ, $\mathrm{H}_{1}=\mathbf{1 2 5}$}

Similar to the experiments in [5], we set $H_{1}$ to 125 to create a more deceiving domain instance: the actions on the suboptimal peak have higher fitness and they are thus more likely to be selected. 


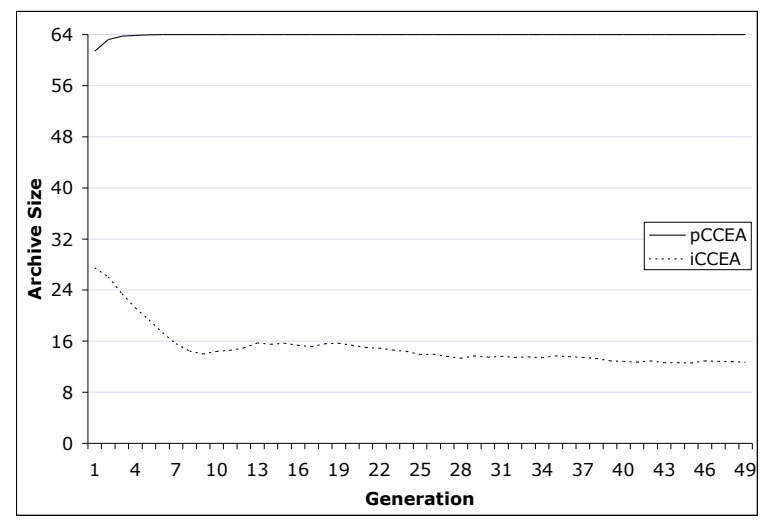

Figure 3: Average archive size for the iCCEA and the pCCEA algorithms in the OneRidge domain

Tables 3-4 present the results of the four methods in the MTQ and SMTQ domain instances with $H_{1}=125$. The results are consistent with the ones in Section 4.1: iCCEA is always better than CCCEA, which is in turn always better than pCCEA (with confidence 95\%). The rCCEA method is worst: it finds the global optimum in only $16 \%$ of the runs in both the MTQ and the SMTQ domain $\left(H_{1}=125\right)$. As before, iCCEA requires significantly fewer evaluations than the other methods. The average archive size is slightly higher (statistically significantly higher for iCCEA) than in the case of $H_{1}=50$, but it follows the same trend as that shown in Figure 2.

\subsection{Experiment 3: The OneRidge Domain}

Our last experiment examined the performance of the search methods in the OneRidge domain proposed in [24]. The OneRidge domain is defined as:

$$
\text { OneRidge }(x, y) \leftarrow 1+2 * \min (x, y)-\max (x, y)
$$

where $x$ and $y$ range between 0 and 1. OneRidge is particularly difficult for concurrent learners because it contains a very large number of Nash equilibria: for any value $v$ between 0 and $1,(v, v)$ is a Nash equilibrium. This implies that for almost any Nash equilibrium (except for the global optimum $(1,1)$ ) there are an infinite number of better Nash equilibria that are infinitesimally close; unfortunately, both agents need to concurrently change their actions for the team to advance to these better solutions. To better study the algorithms' capacity to follow this ridge to the global optimum, we randomly initialized the populations of actions for each agent to only values smaller than 0.5 .

If SMTQ adds more non-linear interactions among the agents, OneRidge goes even further. As a consequence, the methods have a very different ranking based on their performance in this domain (as shown in Table 5). cCCEA performs best, followed in order by rCCEA, iCCEA, and finally pCCEA; there are statistically significant differences among all the methods.

This poor performance of PCCEA and iCCEA seems unexpected at first. To shed more light onto the behavior of the two algorithms, we plotted the average archive size for PCCEA and iCCEA in Figure 3. As expected, the Pareto frontier in the OneRidge domain makes pCCEA think that every possible action is interesting and needs to be added to the archive; consequently, the size of pCCEA's archive is close to 64 even in the very first generations, and so learning stagnates. Unlike MTQ and SMTQ, it is relatively easy in the OneRidge domain to improve upon a joint action by small variations in
Table 3: Results of the four methods in the MTQ domain instance with $\mathrm{H}_{1}=125$

\begin{tabular}{lcccc} 
Method & $\begin{array}{c}\text { First } \\
\text { Quartile }\end{array}$ & Median & $\begin{array}{c}\text { Third } \\
\text { Quartile }\end{array}$ & $\begin{array}{c}\text { Average } \\
\text { \# Evals }\end{array}$ \\
\hline pCCEA & 142.31862 & 149.03314 & 149.83407 & 51200 \\
cCCEA & 125 & 149.99974 & 149.99998 & 51200 \\
iCCEA & 125 & 149.99994 & 149.99998 & 11277.47 \\
rCCEA & 125 & 125 & 125 & 19200
\end{tabular}

Table 4: Results of the four methods in the SMTQ domain instance with $\mathrm{H}_{1}=125$

\begin{tabular}{ccccc} 
Method & $\begin{array}{c}\text { First } \\
\text { Quartile }\end{array}$ & Median & $\begin{array}{c}\text { Third } \\
\text { Quartile }\end{array}$ & $\begin{array}{c}\text { Average } \\
\text { \# Evals }\end{array}$ \\
\hline pCCEA & 125 & 145.66753 & 149.38516 & 51200 \\
cCCEA & 125 & 149.99977 & 149.99998 & 51200 \\
iCCEA & 125 & 149.99995 & 149.99998 & 11406.65 \\
rCCEA & 125 & 125 & 125 & 19200
\end{tabular}

Table 5: Results of the four methods in the OneRidge problem domain

\begin{tabular}{|c|c|c|c|c|}
\hline Method & $\begin{array}{c}\text { First } \\
\text { Quartile }\end{array}$ & Median & $\begin{array}{c}\text { Third } \\
\text { Quartile }\end{array}$ & $\begin{array}{c}\text { Average } \\
\text { \# Evals }\end{array}$ \\
\hline pCCEA & 1.45762 & 1.47224 & 1.48298 & 51200 \\
\hline CCCEA & 1.89700 & 1.91584 & 1.93125 & 51200 \\
\hline iCCEA & 1.50217 & 1.51778 & 1.53398 & 21545.3 \\
\hline rCCEA & 1.82785 & 1.84294 & 1.86300 & 19200 \\
\hline
\end{tabular}

the actions chosen by each agents. As a consequence, both cCCEA and rCCEA are able to improve until outperforming pCCEA.

The poor performance of iCCEA in this domain has a slightly different cause: the archive mechanism was designed to inform the concurrent learning processes of multiple Nash equilibria that are surrounded by large basins of attraction which cannot be avoided by small variations in actions. Given that OneRidge has no such equilibria, the archives serve little purpose, and they instead act to slow the optimization process by reducing the random exploration of the space. As shown in Figure 3, the average archive size of iCCEA is higher than MaxEvals (which was set to 5), and thus actions are not evaluated when in combination with random actions from the other population.

Revised Experiment. To test this hypothesis, we restricted the maximum archive size of iCCEA to only one action (MaxArchiveSize $=1$ in iCCEA-Archive-Selection), and we performed another 250 runs in the OneRidge domain. The median performance of the algorithm was 1.84439 , with a first quartile of 1.82448 and a third quartile of 1.86095 . This is indistinguishable from the performance of the rCCEA method in Table 5. We further doubled the maximum number of generations for the iCCEA algorithm with a maximum archive size of 1 (this setting still involved only around $40 \%$ of the budget used by CCCEA and PCCEA), and we ran it another 250 times. The global optimum was consistently and precisely found in all of them. This is significantly better than all other algorithms we tested in this domain. 


\section{DISCUSSION AND CONCLUSIONS}

It is rational for learning agents to explore those actions that are rewarded better. However, each agent's experimentation with a particular action directly affects the way the other agents perceive the search space. Therefore, we argue that agents may benefit from also exploring those actions that inform their teammates about the structure of the search space. We suggest that cooperating agents should be altruistic: the entire team may benefit if each agent helps its teammates to rank their actions better. We demonstrated this idea in a new cooperative coevolutionary algorithm, iCCEA, which requires significantly fewer evaluations to outperform other cooperative multiagent learning methods on our test problems. We also noted that too much altruism may hurt performance: an agent may waste resources when attempting to inform its teammates about the many Nash equilibria in the space. Restricting the number of informative actions an agent may choose solved the problem in our simple experiments, but we are exploring alternatives that can automatically balance the information provided to other learning agents with the desirability of searching for optimal joint actions. Future work will also examine formal models and guarantees for concurrent learners employing informative actions, as well as demonstrate such methods in complex multiagent domains.

\section{REFERENCES}

[1] Proceedings of the Eighteenth International Joint Conference on Artificial Intelligence (IJCAI), Acapulco, Mexico, 2003. Morgan Kaufmann.

[2] T. Bäck, D. Fogel, and Z. Michalewicz. Handbook of Evolutionary Computation. Oxford University Press, 1997.

[3] R. Becker, S. Zilberstein, V. Lesser, and C. V. Goldman. Solving Transition Independent Decentralized Markov Decision Processes. Journal of Artificial Intelligence Research, 22:423-455, December 2004.

[4] R. Brafman and M. Tennenholtz. Efficient learning equilibrium. In Advances in Neural Information Processing Systems (NIPS-2002), 2002.

[5] A. Bucci and J. Pollack. On identifying global optima in cooperative coevolution. In Hans-Georg Beyer et al. [10], pages 539-544.

[6] L. Bull. Evolutionary computing in multi-agent environments: Partners. In T. Back, editor, Proceedings of the Seventh International Conference on Genetic Algorithms, pages 370-377. Morgan Kaufmann, 1997.

[7] L. Bull. Evolutionary computing in multi-agent environments: Operators. In D. W. V W Porto, N Saravanan and A. E. Eiben, editors, Proceedings of the Seventh Annual Conference on Evolutionary Programming, pages 43-52. Springer Verlag, 1998.

[8] C. Claus and C. Boutilier. The dynamics of reinforcement learning in cooperative multiagent systems. In Proceedings of National Conference on Artificial Intelligence (AAAI-98), pages 746-752, 1998.

[9] K. De Jong. Evolutionary Computation. The MIT Press, 2006.

[10] Hans-Georg Beyer et al., editor. Proceedings of the Genetic and Evolutionary Computation Conference (GECCO) 2005 ACM, 2005.

[11] E. Hansen, D. Bernstein, and S. Zilberstein. Dynamic programming for partially observable stochastic games. In Proceedings of the Nineteenth National Conference on Artificial Intelligence, 2004.
[12] P. Husbands and F. Mill. Simulated coevolution as the mechanism for emergent planning and scheduling. In R. Belew and L. Booker, editors, Proceedings of the Fourth International Conference on Genetic Algorithms, pages 264-270. Morgan Kaufmann, 1991.

[13] Kalyanmoy Deb et al., editor. Proceedings of the Genetic and Evolutionary Computation Conference (GECCO) 2004. Springer, 2004.

[14] S. Kapetanakis and D. Kudenko. Reinforcement learning of coordination in cooperative multi-agent systems. In Proceedings of the Nineteenth National Conference on Artificial Intelligence (AAAI-02), 2002.

[15] M. Lauer and M. Riedmiller. An algorithm for distributed reinforcement learning in cooperative multi-agent systems. In Proceedings of the Seventeenth International Conference on Machine Learning, pages 535-542. Morgan Kaufmann, 2000.

[16] M. I. Lichbach. The cooperator's dilemma. University of Michigan Press, 1996.

[17] S. Luke. ECJ 13: A Java EC research system. Available at http://cs.gmu.edu/ eclab/projects/ecj/, 2005.

[18] R. Nair, M. Tambe, M. Yokoo, D. Pynadath, and S. Marsella. Taming decentralized POMDPs: Towards efficient policy computation for multiagent settings. In Proceedings of the Eighteenth International Joint Conference on Artificial Intelligence (IJCAI), 2003.

[19] L. Panait and S. Luke. Cooperative multi-agent learning: The state of the art. Autonomous Agents and Multi-Agent Systems, 11(3), 2005.

[20] L. Panait and S. Luke. Time-dependent collaboration schemes for cooperative coevolutionary algorithms. In Proceedings of the 2005 AAAI Fall Symposium on Coevolutionary and Coadaptive Systems, 2005.

[21] L. Panait, R. P. Wiegand, and S. Luke. Improving coevolutionary search for optimal multiagent behaviors. In Proceedings of the Eighteenth International Joint Conference on Artificial Intelligence (IJCAI) [1], pages 653-658.

[22] L. Panait, R. P. Wiegand, and S. Luke. A sensitivity analysis of a cooperative coevolutionary algorithm biased for optimization. In Kalyanmoy Deb et al. [13], page (to appear).

[23] L. Panait, R. P. Wiegand, and S. Luke. A visual demonstration of convergence properties of cooperative coevolution. In Parallel Problem Solving from Nature PPSN-2004. Springer, 2004.

[24] E. Popovici and K. D. Jong. Understanding cooperative co-evolutionary dynamics via simple fitness landscapes. In Hans-Georg Beyer et al. [10], pages 507-514.

[25] M. Potter. The Design and Analysis of a Computational Model of Cooperative CoEvolution. $\mathrm{PhD}$ thesis, George Mason University, Fairfax, Virginia, 1997.

[26] Y. Shoham, R. Powers, and T. Grenager. On the agenda(s) of research on multi-agent learning. In Proceedings of Artificial Multiagent Learning. Papers from the 2004 AAAI Fall Symposium. Technical Report FS-04-02, 2004.

[27] D. Szer, F. Charpillet, and S. Zilberstein. MAA*: A heuristic search algorithm for solving decentralized POMDPs. In Proceedings of the Twenty First Conference on Uncertainty in Artificial Intelligence, 2005.

[28] R. P. Wiegand. Analysis of Cooperative Coevolutionary Algorithms. PhD thesis, Department of Computer Science, George Mason University, 2003. 
[29] R. P. Wiegand, W. Liles, and K. De Jong. An empirical analysis of collaboration methods in cooperative coevolutionary algorithms. In Proceedings of the Genetic and Evolutionary Computation Conference (GECCO), pages 1235-1242, 2001. 